\title{
Prolapso de gordura orbitária bilateral associado a pterígio: relato de caso
}

\author{
Bilateralorbital fat prolapse associated with pterygium:case report
}

\author{
Vanderson Glerian Dias ${ }^{1}$ \\ Dino de Aguiar Cintra ${ }^{2}$ \\ Edmilson Gigante ${ }^{3}$
}

Trabalho realizado na Universidade do Oeste Paulista - UNOESTE - Presidente Prudente (SP) - Brasil.

${ }^{1}$ Colaborador de Pesquisa do Hospital de Olhos Redentora - São José do Rio Preto (SP) - Brasil.

${ }^{2}$ Responsável pelo Serviço de Plástica e Vias Lacrimais do Hospital Universitário da Universidade do Oeste Paulista - UNOESTE - Presidente Prudente (SP) - Brasil.

${ }^{3}$ Oftalmologista Chefe do Serviço de Residência Médica em Oftalmologista do Hospital Universitário da UNOESTE - Presidente Prudente (SP) - Brasil.

Endereço para correspondência: Vanderson Glerian Dias. Rua Guaporé, 432 - Catanduva (SP) CEP 15804-105 E-mail: glerian@globo.com

Recebido para publicação em 14.01.2005

Versão revisada recebida em 04.12 .2005

Aprovação em 13.12.2005

\section{RESUMO}

O prolapso de gordura orbitária é entidade benigna incomum que pode causar defeitos estéticos. Os autores apresentam uma paciente de 63 anos com prolapso de gordura orbitária temporal bilateral associado a pterígio medial bilateral, ressaltando aspectos clínicos e cirúrgicos. Discute-se a importância do diagnóstico clínico e de imagem, além da comprovação histopatológica da lesão.

Descritores: Tecido adiposo; Doenças orbitárias/diagnóstico; Prolapso; Doenças orbitárias/ cirurgia; Pterígio/cirurgia; Relatos de casos [Tipo de publicação]

\section{INTRODUÇ̃̃O}

O tecido de gordura envolve todas as estruturas orbitárias, fornecendo sustentação para o globo ocular e facilitando a movimentação dos olhos. O septo orbitário tem a função de conter a gordura da órbita e a cápsula de Tenon isola a esclera e os músculos da gordura orbitária ${ }^{(1-2)}$. O prolapso da gordura orbitária (PGO) ocorre quando há defeito na cápsula de Tenon e um afinamento do septo orbitário ${ }^{(1,3-4)}$. Esta alteração pode se manifestar em qualquer localização do globo, porém é mais freqüente na região temporal.

O PGO é uma entidade clínica espontânea, benigna, que surge na idade adulta e pode ser facilmente confundida com dermolipoma ${ }^{(1,3-5)}$. Clinicamente essas patologias são vistas como lesões de coloração levemente amarelada, homogênea, coberta por conjuntiva de superfície lisa, contendo poucos vasos e sem sinais inflamatórios ${ }^{(1)}$. Devido à dificuldade clínica do diagnóstico diferencial, faz-se necessária comprovação através de exame histopatológico da lesão.

O objetivo deste trabalho é apresentar um caso de PGO temporal bilateral associado a pterígio medial bilateral, diagnosticado e tratado no Hospital Universitário da Universidade do Oeste Paulista - UNOESTE.

\section{RELATO DO CASO}

MJP, sexo feminino, 63 anos, raça negra, procurou o serviço de oftalmologia do Hospital Universitário da Universidade do Oeste Paulista - UNOESTE, de Presidente Prudente - SP, em junho de 2004, relatando aparecimento de caroços nos olhos há mais de cinco anos. Afirmava progressão lenta e assintomática.

Em relação aos antecedentes pessoais apresentava diabetes mellitus tipo II em uso de Glibenclamida ${ }^{\circledR}$, hipertensão arterial sistêmica em uso de Captopril $^{\circledR}$, além de ter feito cirurgia de facectomia com implante de LIO no olho esquerdo há um ano em outro serviço.

Exame oftalmológico: acuidade visual corrigida de 0,9 em ambos os 
olhos (AO) e J1 para perto em AO com adição de +2,50 esférico; pressão ocular de $14 \mathrm{mmHg}$ em AO; motilidade ocular normal; fundoscopia normal. Ao exame externo, assim como à biomicroscopia, presença de cistos temporais em AO, de consistência amolecida, coloração esbranquiçada, não aderentes à conjuntiva nem à esclera, além de halo senil corneano em AO, pterígio medial grau I elevado no olho direito (OD) e pterígio medial grau II no olho esquerdo (Figuras 1 e 2).

No primeiro ato cirúrgico realizou-se exérese simples de pterígio em OD sendo feitas duas suturas conjuntivais com Vicryl $^{\circledR}$ 8-0, deixando pequena região de esclera nua perilimbar, além da exérese simples do suposto cisto, suturandose a conjuntiva também com Vicryl ${ }^{\circledR}$ 8-0. Após um mês da primeira intervenção, os mesmos procedimentos foram realizados no olho contra-lateral (Figura 3).

O resultado do exame histopatológico das lesões císticas comprovou ser tecido formado por adipócitos maduros e congestão vascular, sem outras particularidades.

\section{DISCUSSÃO}

O PGO é facilmente confundido com tumores do tecido gorduroso, como lipomas e lipossarcomas; coristomas (lesões presentes desde o nascimento, com pequeno ou sem potencial

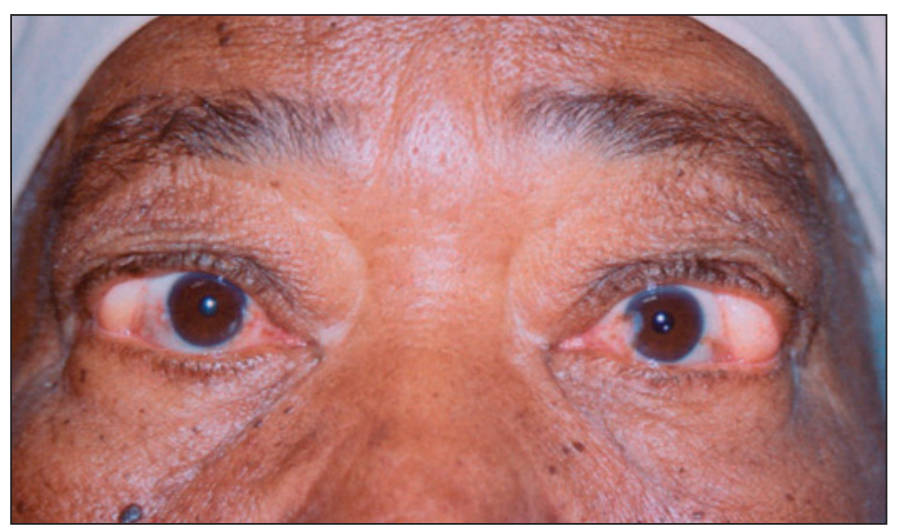

Figura 1 - Aspecto macroscópico das lesões

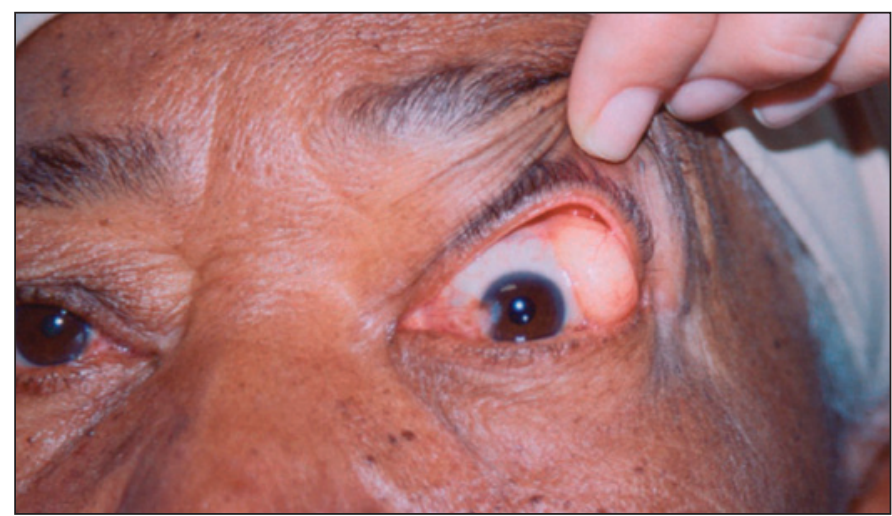

Figura 2 - Aspecto macroscópico do prolapso de gordura orbitária em olho esquerdo para o crescimento, formadas por tecidos normais que, habitualmente, não são encontrados na região envolvida); ptose ou tumor de glândula lacrimal; tumores linfóides; além de outros tumores da superfície ocular ${ }^{(1,5-7)}$. O diagnóstico diferencial mais importante é com o dermolipoma que é um tipo de coristoma. O coristoma ósseo episcleral, mesmo sendo raro, também deve ser incluído como possível patologia, até a comprovação da suspeita diagnóstica ${ }^{(8)}$.

Devido a possível confusão diagnóstica e mau planejamento cirúrgico diante de tumores da superfície ocular, os exames de imagem podem auxiliar a elucidação da doença na tentativa de excluir algumas patologias. As lesões ósseas, ou com componentes ósseos, são radiopacas e podem ser, frequentemente, demonstradas no exame radiológico ${ }^{(9)}$. O uso da biomicroscopia ultra-sônica pode ser feito na grande maioria das lesões superficiais do olho com boa sensibilidade e especificidade, até mesmo em tumores pequenos ${ }^{(8)}$. A ultra-sonografia de alta frequiência ou a injeção de indocianina verde podem ser utilizadas com intuito de diagnosticar as margens de segurança no caso de formações císticas ${ }^{(10-12)}$. Achados de tomografia computadorizada também podem ser incluídos no arsenal clínico com intuito de fechar o diagnóstico no préoperatório $^{(13)}$. Neste relato de caso, no entanto, não foi realizado nenhum estudo de imagem devido à facilidade do diagnóstico clínico das lesões.

Um trabalho avaliou 10 pacientes com PGO e relatou a associação com cisto pilonidal e triquíase ${ }^{(1)}$. Em nosso relato houve associação de PGO com pterígio medial bilateral. Não se pode dizer, entretanto, que alterações inflamatórias possam estar fisiologicamente e/ou patologicamente associadas ao PGO já que a causa da fragilidade das estruturas de contenção da gordura orbitária não é totalmente conhecida. Essas associações possivelmente sejam ocasionais e dependendo, portanto, de outros fatores, como o genético e/ou ambiental, no caso do pterígio; inflamatório, no caso do cisto pilonidal e, provavelmente; infeccioso ou estrutural, no caso da triquíase.

Na maioria dos casos o aparecimento do PGO é unilateral, em pacientes com idade acima de 30 anos e em região lateral, espontâneo, após cirurgia ou trauma. Porém alguns relatos indicam o comprometimento bilateral, medial e em pacientes

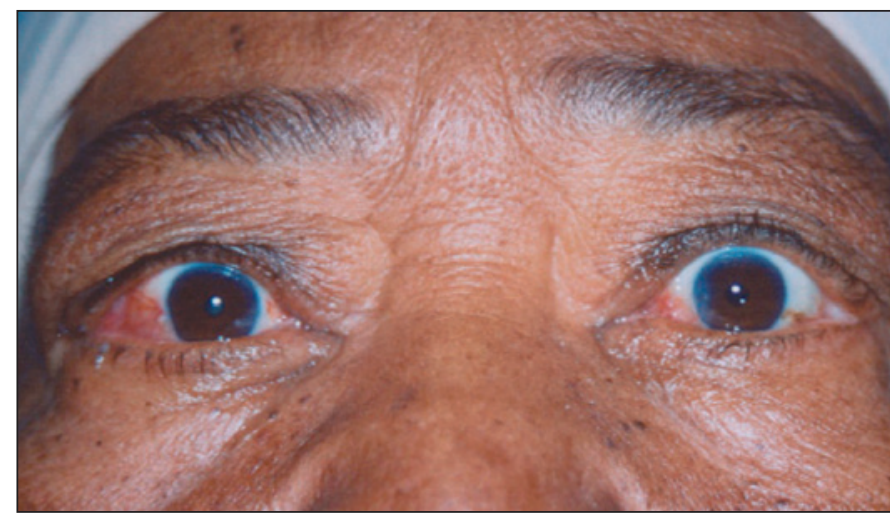

Figura 3 - Aspecto macroscópico após um mês da última intervenção cirúrgica 
jovens ${ }^{(7,14)}$. Neste trabalho a paciente relatou início espontâneo, mais ou menos simétrico, sem relação com trauma ou cirurgia ocular realizada antes da presença das lesões.

A queixa do paciente normalmente é a lesão, em relação a sua função antiestética, como neste relato, porém, desconforto ocular e outros sintomas também são descritos ${ }^{(1,14)}$.

Pacientes com PGO não necessitam de procedimento cirúrgico. Muitas vezes é realizado por motivo estético e a conduta cirúrgica mais correta é a retirada da porção anterior aparente, sem o aprofundamento na órbita ${ }^{(1,15)}$. Não obstante, pode-se utilizar outras técnicas cirúrgicas como a transposição dos pedículos de gordura orbitária para dentro da bolsa subperiosteal ${ }^{(16)}$ ou utilizando-se da retirada da gordura anterior com fixação escleral da conjuntiva aproximadamente 8 a $10 \mathrm{~mm}$ posterior ao limbo corneano, através de 4 a 7 suturas com Vicryl 6-0 $0^{(17)}$.

Em relação ao exame histopatológico, não há dúvida em sua comprovação diagnóstica já que o resultado da exérese de PGO demonstra lesão composta por adipócitos maduros normais. Além disso, a diferenciação clínica é possível na maioria dos casos dessa entidade patológica.

\section{ABSTRACT}

Orbital fat prolapse is an uncommon benign entity that can cause esthetic defects. The authors present a 63-year-old patient with bilateral temporal orbital fat prolapse associated with a bilateral nasal pterygium. Clinical and surgical features are described. The clinical, imaging and histopathological diagnoses are discussed.

Keywords: Adipose tissue; Orbital diseases/diagnosis; Prolapse; Orbital disease/surgeries; Pterygium/surgery; Case reports [Publication type]

\section{REFERÊNCIAS}

1. Ferraz LCB, Schellini SA, Wludarski SCL, Marques MEA, Moraes-Silva MRB. Dermolipoma e prolapso de gordura orbital: duas entidades distintas. Arq Bras Oftalmol. 2002;65(3):327-31

2. Souza-Dias CR. Anatomia dos Músculos Oculomotores, suas Fáscias e Inervação. In: Souza-Dias CR. editor. Estrabismo. Rio de Janeiro: Cultura Médica; 1999. p.5.

3. Offret H, Labetoulle M, Frau E. [Subconjunctival adipose hernias of the orbit]. J Fr Ophtalmol. 1997;20(8):614-8. French.

4. Jordan DR, Tse DT. Herniated orbital fat. Can J Ophthalmol. 1987;22(3):173-7.

5. Liao SB, Ku WC, Song HS, Lin CY. Spontaneous subconjunctival orbital fat prolapse: report of three cases. Chang Gung Med J. 2001;24(6):399-403.

6. Spencer W, Zimmerman LE. Conjunciva. In: Spencer WH. Ophthalmic pathology: an atlas and textbook. 3rd ed. Philadelphia: W.B. Saunders; 1985-1986. p.117-27.

7. Monner J, Benito JR, Zayuelas J, Paloma V, Castro V, Serra JM. Transconjunctival herniation of orbital fat. Ann Plast Surg. 1998;41(6):658-61.

8. Ferraz LCB, Hoyama E, Schellini SA, Wludarski SCL, Marques MEA. Coristoma ósseo episcleral: relato de caso. Arq Bras Oftalmol. 2003;66(5):667-9.

9. Beckman H, Sugar HS. Episcleral osseous choristoma - report of two cases. Arch Ophthalmol. 1964;71:377-8.

10. Dias VG, Martins MP, Bezzon AKT, Aguni JS, Cavalheiro R. Cisto de inclusão conjuntival gigante associado a pterígio: relato de caso. Arq Bras Oftalmol. 2004;67(5):831-3.

11. Finger PT. Giant Conjunctival inclusion cysts [text on the Internet]. New York: Eye Cancer Network. Case 6. [cited 2000 Nov 21]. Available from: http: //www.eyecancer.com/Content.aspx?sSection=Research\&sSubSection=Content \&sPage=Research.ascx \&nID=12\&Research=Eye+Cancer+Network+Case+\%236 $\% 3 \mathrm{a}+$ Giant+Conjunctival++Inclusion+Cysts

12. Kobayashi A, Saeki A, Nishimura A, Segawa Y, Shirao Y, Kawasaki K. Visualization of conjunctival cyst by indocyanine green. Am J Ophthalmol. 2002;133(6):827-8

13. Glover AT, Grove AS Jr. Subconjunctival orbital fat prolapse. Ophthal Plast Reconstr Surg. 1987;3(2):83-6

14. McNab AA. Subconjunctival fat prolapse. Aust N Z J Ophthalmol. 1999;27(1): 33-6.

15. Alves MR, Kara-José N. Conjuntiva cirúrgica. São Paulo: Rocca; 1999. p.255.

16. Goldberg RA. Transconjunctival orbital fat repositioning: transposition of orbital fat pedicles into a subperiosteal pocket. Plast Reconstr Surg. 2000;105(2): 743-8; discussion 749-51.

17. Otaka I, Kyu N. A new surgical technique for the management of orbital fat prolapse. Am J Ophthalmol. 2001;131(2):267-9.

\section{Congresso Internacional da Sociedade Brasileira de Oftalmologia}

20 a 22 de Julho de 2006

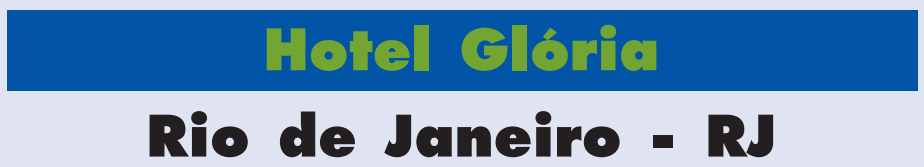

INFORMAÇÕES: Tel.: (21) 2557-7728

E-mail: sbo@sboportal.org.br 